\title{
Isolation And Characterization Of 45 SNP Markers In Triplophysa Tenuis
}

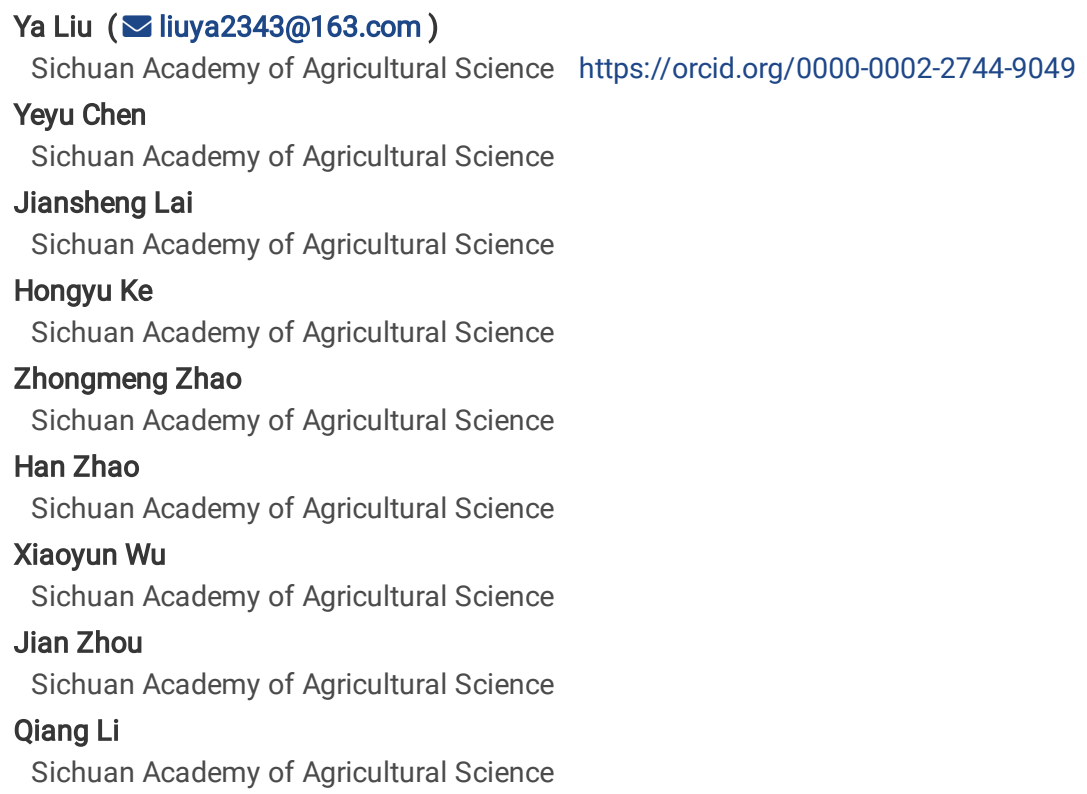

\section{Research Article}

Keywords: Triplophysa tenuis, SNP marker, Genetic diversity analysis, RAD-seq

Posted Date: June 14th, 2021

DOI: https://doi.org/10.21203/rs.3.rs-551660/v1

License: (ㅇ) (1) This work is licensed under a Creative Commons Attribution 4.0 International License. Read Full License

Version of Record: A version of this preprint was published at Conservation Genetics Resources on February 22nd, 2022. See the published version at https://doi.org/10.1007/s12686-022-01254-7. 


\section{Abstract}

Triplophysa tenuis is an endemic species to China, which mainly distributed in Xinjiang and Gansu province. Effective conservation and management of this species is limited by insufficient molecular markers. In the present study, we reported the isolation and characterization of 45 SNP markers in $T$. tenuis. The minor allele frequency ranged from 0.046 to 0.500 , and the observed and expected heterozygosities ranged from 0.061 to 0.667 and 0.088 to 0.508 , respectively. Polymorphic information content ranged from 0.083 to 0.375 . Among these SNPs, three loci showed significant departures from the Hardy-Weinberg equilibrium. The novel polymorphic SNPs will be helpful for the future study on genetic management and population conservation for this species.

\section{Main Text}

Triplophysa tenuis is distributed both in the Bosten lake and Tarim river systems in the south of Xinjiang and in the Shule river and Ruoshui river in the Hexi corridor of Gansu province (Zhu 1989). Although T. tenuis has not yet been classified as an endangered species, the fish species in Gansu province showed an obvious decreasing trend, especially the degradation of endemic fish (Wang et al. 2015). At present, the reports on T. tenuis are mainly in the aspect of biological characteristics (Chen et al. 2017, Yao et al. 2018). There are very few reports about molecular markers in this species. Genetic markers that are currently available for T. tenuis have mostly focused on mitochondrial DNA (Wang et al. 2015, Wang et al. 2016). Single-nucleotide polymorphism (SNP) markers are useful in population genetic studies because of their co-dominance, high levels of polymorphism, low cost and wide distribution (Vignal et al. 2002, Wang et al. 2015, Blanc-Jolivet et al. 2017). In the present study, we developed and characterized 45 SNP markers for the first time in T. tenuis. It is expected that these results will contribute to population conservation, genetic management and construction of genetic linkage map for this species.

In this study, restriction-site associated DNA sequencing (RAD-seq) was used to isolate and characterize SNP markers. A total of eighty potential SNP loci were selected for primer design using Primer 5.0. Fin clips of T. tenuis were collected from 33 individuals of the Shule river (Gansu, China). Genomic DNA was extracted using the cetyltrimethylammonium bromide (CTAB) method (Doyle 1987) and was stored at $-20^{\circ} \mathrm{C}$.

PCR amplifications were performed in $30 \mu \mathrm{L}$ volumes containing $1 \mu \mathrm{L}$ of genomic DNA, $14 \mu \mathrm{L}$ of Premix Taq ( $2 \times$ Taq Plus MasterMix, CWBIO), $1 \mu \mathrm{L}$ of each gene primer and $13 \mu \mathrm{L}$ of PCR-grade water. The PCR programme was $94^{\circ} \mathrm{C}$ for $5 \mathrm{~min}$, then 32 cycles at $94^{\circ} \mathrm{C}$ for $30 \mathrm{~s}$, annealing for $30 \mathrm{~s}$ (for annealing temperatures of each primer pair, Table 1), $72^{\circ} \mathrm{C}$ for $45 \mathrm{~s}$, and one cycle of $72^{\circ} \mathrm{C}$ for 7 min for the final extension. Amplification products were sequenced using Sanger technology. The sequenced fragments were aligned using Vector NTI 10.3.0 (Invitrogen, Carlsbad, CA, USA) and the genotypes per locus were determined by BioEdit according to the peaks of each base. The minor allele frequency (MAF), observed heterozygosities (Ho), expected heterozygosities $(\mathrm{He})$, polymorphism information content (PIC) and P value representing the deviations from Hardy-Weinberg equilibrium (HWE) were calculated using Cervus 3.0 (Kalinowski et al. 2007).

A total of 45 loci were found to be polymorphic and showed bi-allelic in 33 individuals of $T$. tenuis. The minor allele frequency ranged from 0.046 to 0.500 (Table 1). The observed heterozygosity varied from 0.061 to 0.667 , while the expected heterozygosity ranged from 0.088 to 0.508 . Polymorphic information content ranged from 0.083 to 0.375 . Only three loci showed significant deviations from the HWE after Bonferroni correction. As far as we know, this is the first report of SNP identification in T. tenuis, which will be valuable tool for population conservation in this species. 
Table 1

Characterization of 45 SNPs in T. tenuis

\begin{tabular}{|c|c|c|c|c|c|c|c|c|c|c|c|}
\hline $\begin{array}{l}\text { Primer } \\
\text { ID }\end{array}$ & Primer sequences $\left(5^{\prime}-3^{\prime}\right)$ & $\begin{array}{l}\text { Fragment } \\
\text { size (bp) }\end{array}$ & Locus ID & $\begin{array}{l}\text { SNP } \\
\text { type }\end{array}$ & $\begin{array}{l}\text { SNP } \\
\text { position }\end{array}$ & $\begin{array}{l}\mathrm{T}_{\mathrm{a}} \\
\left({ }^{\circ} \mathrm{C}\right)\end{array}$ & MAF & Ho & $\mathrm{He}$ & PIC & HWEP \\
\hline \multirow[t]{2}{*}{ TtSNP1 } & F:TCACAGACGTACATTTCCCATA & 248 & TtSNP1-1 & $\mathrm{C} / \mathrm{T}$ & 128 & 48 & 0.152 & 0.182 & 0.261 & 0.224 & NS \\
\hline & R:ATTCAAACACTGAGTGCTTTTT & & TtSNP1-2 & $A / G$ & 147 & & 0.273 & 0.242 & 0.403 & 0.318 & NS \\
\hline \multirow[t]{3}{*}{ TtSNP2 } & F:GCCTGTTTGTGTGTTTAACCTG & 356 & TtSNP2-1 & $\mathrm{C} / \mathrm{G}$ & 177 & 51 & 0.182 & 0.303 & 0.302 & 0.253 & NS \\
\hline & R:ctagaaacctgccagattcct & & TtSNP2-2 & $\mathrm{A} / \mathrm{T}$ & 197 & & 0.197 & 0.333 & 0.321 & 0.266 & NS \\
\hline & & & TtSNP2-3 & $\mathrm{G} / \mathrm{T}$ & 253 & & 0.485 & 0.667 & 0.507 & 0.375 & NS \\
\hline \multirow[t]{5}{*}{ TtSNP3 } & F:CAATAGAAGTCAATGGGGATCG & 340 & TtSNP3-1 & $\mathrm{C} / \mathrm{G}$ & 186 & 50 & 0.106 & 0.212 & 0.193 & 0.172 & NS \\
\hline & R:ACAGACCACATAGATCATCCAT & & TtSNP3-2 & $\mathrm{C} / \mathrm{T}$ & 187 & & 0.318 & 0.394 & 0.441 & 0.340 & NS \\
\hline & & & TtSNP3-3 & $A / G$ & 256 & & 0.303 & 0.424 & 0.429 & 0.333 & NS \\
\hline & & & TtSNP3-4 & $\mathrm{A} / \mathrm{T}$ & 309 & & 0.318 & 0.455 & 0.441 & 0.340 & NS \\
\hline & & & TtSNP3-5 & $\mathrm{C} / \mathrm{T}$ & 314 & & 0.121 & 0.182 & 0.216 & 0.190 & NS \\
\hline \multirow[t]{7}{*}{ TtSNP4 } & F:GCTTTCCTGTAGCTCAGTTGTA & 371 & TtSNP4-1 & $A / G$ & 92 & 51 & 0.394 & 0.364 & 0.485 & 0.363 & NS \\
\hline & R:ATAAGGGACTTTATTGCGTTCATGC & & TtSNP4-2 & $\mathrm{A} / \mathrm{T}$ & 93 & & 0.424 & 0.364 & 0.496 & 0.369 & NS \\
\hline & & & TtSNP4-3 & $\mathrm{G} / \mathrm{T}$ & 162 & & 0.091 & 0.061 & 0.168 & 0.152 & * \\
\hline & & & TtSNP4-4 & $\mathrm{A} / \mathrm{T}$ & 183 & & 0.364 & 0.424 & 0.470 & 0.356 & NS \\
\hline & & & TtSNP4-5 & $\mathrm{C} / \mathrm{T}$ & 313 & & 0.394 & 0.606 & 0.485 & 0.363 & NS \\
\hline & & & TtSNP4-6 & $\mathrm{C} / \mathrm{T}$ & 322 & & 0.136 & 0.273 & 0.239 & 0.208 & NS \\
\hline & & & TtSNP4-7 & $\mathrm{A} / \mathrm{T}$ & 326 & & 0.379 & 0.576 & 0.478 & 0.360 & NS \\
\hline \multirow[t]{5}{*}{ TtSNP5 } & F:TTCCTAACCAGGTCAAAAGTAA & 319 & TtSNP5-1 & $\mathrm{A} / \mathrm{G}$ & 103 & 48 & 0.273 & 0.121 & 0.403 & 0.318 & $\star *$ \\
\hline & R:AGCTTTGAAGCATTTACAGTCA & & TtSNP5-2 & $A / G$ & 155 & & 0.485 & 0.364 & 0.507 & 0.375 & NS \\
\hline & & & TtSNP5-3 & $A / G$ & 251 & & 0.046 & 0.091 & 0.088 & 0.083 & NS \\
\hline & & & TtSNP5-4 & $\mathrm{A} / \mathrm{T}$ & 278 & & 0.227 & 0.333 & 0.357 & 0.290 & NS \\
\hline & & & TtSNP5-5 & $A / G$ & 285 & & 0.197 & 0.333 & 0.321 & 0.266 & NS \\
\hline \multirow[t]{2}{*}{ TtSNP6 } & F:CCССАТССАТTCAGCАTСТСT & 251 & TtSNP6-1 & $\mathrm{C} / \mathrm{G}$ & 212 & 51 & 0.212 & 0.303 & 0.339 & 0.278 & NS \\
\hline & R:CTATTGCTAATTCGAGAGGGTG & & & & & & & & & & \\
\hline \multirow[t]{2}{*}{ TtSNP7 } & F:CAAAATGAAACCAAATCCTGCC & 329 & TtSNP7-1 & $A / G$ & 254 & 49 & 0.091 & 0.182 & 0.168 & 0.152 & NS \\
\hline & R:GAGGCAAATAAATTAGACCACAAAT & & TtSNP7-2 & $\mathrm{A} / \mathrm{T}$ & 281 & & 0.349 & 0.515 & 0.461 & 0.351 & NS \\
\hline \multirow[t]{7}{*}{ TtSNP8 } & F:GTCGTACCCCAAACAGTTCTG & 324 & TtSNP8-1 & $A / G$ & 122 & 51 & 0.167 & 0.212 & 0.282 & 0.239 & NS \\
\hline & R:AAGATAAGCAGAGAGAACAACAC & & TtSNP8-2 & $\mathrm{C} / \mathrm{T}$ & 142 & & 0.409 & 0.576 & 0.491 & 0.367 & NS \\
\hline & & & TtSNP8-3 & $\mathrm{C} / \mathrm{T}$ & 153 & & 0.303 & 0.364 & 0.429 & 0.333 & NS \\
\hline & & & TtSNP8-4 & $\mathrm{A} / \mathrm{T}$ & 156 & & 0.061 & 0.121 & 0.116 & 0.107 & NS \\
\hline & & & TtSNP8-5 & $\mathrm{C} / \mathrm{T}$ & 193 & & 0.333 & 0.364 & 0.451 & 0.346 & NS \\
\hline & & & TtSNP8-6 & $\mathrm{C} / \mathrm{T}$ & 197 & & 0.303 & 0.364 & 0.429 & 0.333 & NS \\
\hline & & & TtSNP8-7 & $\mathrm{A} / \mathrm{G}$ & 224 & & 0.091 & 0.182 & 0.168 & 0.152 & NS \\
\hline \multirow[t]{6}{*}{ TtSNP9 } & F:СТTАССТССТАСТТССТСG & 339 & TtSNP9-1 & $\mathrm{C} / \mathrm{T}$ & 157 & 49 & 0.061 & 0.061 & 0.116 & 0.107 & NS \\
\hline & R:TGTCCTGAAGTTTTGAGCGAA & & TtSNP9-2 & $\mathrm{C} / \mathrm{T}$ & 172 & & 0.273 & 0.424 & 0.403 & 0.318 & NS \\
\hline & & & TtSNP9-3 & $\mathrm{C} / \mathrm{G}$ & 175 & & 0.046 & 0.091 & 0.088 & 0.083 & NS \\
\hline & & & TtSNP9-4 & $\mathrm{G} / \mathrm{T}$ & 183 & & 0.364 & 0.364 & 0.470 & 0.356 & NS \\
\hline & & & TtSNP9-5 & $\mathrm{A} / \mathrm{T}$ & 185 & & 0.136 & 0.152 & 0.239 & 0.208 & NS \\
\hline & & & TtSNP9-6 & $\mathrm{A} / \mathrm{T}$ & 255 & & 0.106 & 0.091 & 0.193 & 0.172 & NS \\
\hline
\end{tabular}




\begin{tabular}{|c|c|c|c|c|c|c|c|c|c|c|c|}
\hline $\begin{array}{l}\text { Primer } \\
\text { ID }\end{array}$ & Primer sequences (5'-3') & $\begin{array}{l}\text { Fragment } \\
\text { size (bp) }\end{array}$ & Locus ID & $\begin{array}{l}\text { SNP } \\
\text { type }\end{array}$ & $\begin{array}{l}\text { SNP } \\
\text { position }\end{array}$ & $\begin{array}{l}\mathrm{T}_{\mathrm{a}} \\
\left({ }^{\circ} \mathrm{C}\right)\end{array}$ & MAF & Ho & $\mathrm{He}$ & PIC & HWEP \\
\hline \multirow[t]{6}{*}{ TtSNP10 } & F:CTACACACTGTTTACCTGGCC & 383 & $\begin{array}{l}\text { TtSNP10- } \\
1\end{array}$ & $\mathrm{~A} / \mathrm{T}$ & 126 & 53 & 0.091 & 0.182 & 0.168 & 0.152 & NS \\
\hline & R:TAATCAGGGGCACTGTGGAAG & & $\begin{array}{l}\text { TtSNP10- } \\
2\end{array}$ & $\mathrm{~A} / \mathrm{C}$ & 132 & & 0.197 & 0.091 & 0.321 & 0.266 & $\star \star$ \\
\hline & & & $\begin{array}{l}\text { TtSNP10- } \\
3\end{array}$ & $\mathrm{C} / \mathrm{T}$ & 195 & & 0.152 & 0.182 & 0.261 & 0.224 & NS \\
\hline & & & $\begin{array}{l}\text { TtSNP10- } \\
4\end{array}$ & $\mathrm{C} / \mathrm{T}$ & 196 & & 0.424 & 0.545 & 0.496 & 0.369 & NS \\
\hline & & & $\begin{array}{l}\text { TtSNP10- } \\
5\end{array}$ & $\mathrm{~A} / \mathrm{C}$ & 229 & & 0.152 & 0.182 & 0.261 & 0.224 & NS \\
\hline & & & $\begin{array}{l}\text { TtSNP10- } \\
6\end{array}$ & $\mathrm{~A} / \mathrm{T}$ & 276 & & 0.197 & 0.091 & 0.321 & 0.266 & *夫 \\
\hline \multirow[t]{2}{*}{ TtSNP11 } & F:TGTATTTGCTTATTTGACACTCAA & 273 & ${ }_{1}^{\text {TtSNP11- }}$ & $\mathrm{A} / \mathrm{T}$ & 218 & 48 & 0.500 & 0.636 & 0.508 & 0.375 & NS \\
\hline & R:TTCTGATGGACTGAGAGATAGA & & & & & & & & & & \\
\hline
\end{tabular}

MAF minor allele frequency, Ho observed heterozygosity, He expected heterozygosity, PIC polymorphism information content, HWEP results for HardyWeinberg Equilibrium text, NS non-significant

$\star \mathrm{P}<0.05 * \star \mathrm{P}<0.01$

\section{Declarations}

Acknowledgements

The authors are grateful to Wenqiong Wu and Siyi Zhu for their assistance with the laboratory work.

Funding

This work was supported by grants from the Project of Investigation on Fishery Resources and Environment of Northwest China, the Project of Sichuan Science and Technology Program (2021YFYZ0015).

Competing Interests

The authors do not have any conflict of interest.

Availability of data and material

The data and material that support the findings of this study are available from the corresponding author upon reasonable request.

Code availability

Not applicable

Authors' contribution

Ya Liu conceived and designed the experiments. Ya Liu wrote the manuscript. Yeyu Chen revised the manuscript. Jiansheng Lai, Xiaoyun Wu and Qiang Li performed the experiments. Hongyu Ke, Zhongmeng Zhao, Han Zhao and Jian Zhou provide a source of this species. Yeyu Chen and Qiang Li provided formal analysis. All authors read and approved the final manuscript.

Ethics approval

All fish handling and experimental procedures were approved by the Animal Care and Use Committee of the Fishery Institute of the Sichuan Academy of Agricultural Sciences, and all animal collection and use protocols were carried out in accordance with the guidelines and regulations for the care and use of laboratory animals at the Fishery Institute of the Sichuan Academy of Agricultural Sciences.

Consent to participate

The authors provide consent to participate.

Consent to publication 
The authors provide consent for publication.

\section{References}

1. Blanc-Jolivet C, Kersten B, Daïnou K, Hardy O, Guichoux E, Del-camp A, Degen B (2017) Development of nuclear SNP markers for genetic tracking of Iroko, Milicia excelsa and Milicia regia. Conserv Genet Res 9:531-533

2. Chen S, Xie C, Li D, Yao N, Ding H, Zhang Z (2017) Length-weight relationships of five Triplophysa species from the northwest of China. J Appl Ichthyol 00:1-3. https://doi.org/10.1111/jai.13432

3. Doyle J (1987) A rapid DNA isolation procedure for small quantities of fresh leaf tissue. Phytochem Bull 19:11-15

4. Kalinowski ST, Taper ML, Marshall TC (2007) Revising how the computer program CERVUS accommodates genotyping error increases success in paternity assignment. Mol Ecol 16:1099-1106. https://doi.10.1111/j.1365-294X.2007.03089.x

5. Vignal A, Milan D, SanCristobal M, Eggen A (2002) A review on SNP and other types of molecular markers and their use in animal genetics. Genet Sel Evol 34:275-305. https://doi.10.1051/gse:2002009

6. Wang L, Huang SQ, Xia JH, Liu P, Wan ZY, Yue GH (2015) Genome-wide discovery of gene-related SNPs in Barramundi Lates calcarifer. Conserv Genet Resour 7:605-608

7. Wang T, Zhang Y, Guan L, Du Y, Lou Z, Jiao W (2015) Current freshwater fish resources and the application of DNA barcoding in species identification in Gansu Province. Biodiversity Science 23:306-313

8. Wang Y, Shen Y, Feng C, Zhao K, Song Z, Zhang Y, Yang L, He S (2016) Mitogenomic perspectives on the origin of Tibetan loaches and their adaptation to high altitude. Sci Rep 6:29690

9. Yao N, Liu J, Ma L, Jin S, Wang S, Chen S, Song Y (2018) Biological Characteristics of Triplophysa bombifrons and Triplophysa tenuis in the upper reaches of Tarim river. Guizhou Agricultural Sciences 46:82-86

10. Zhu S (1989) The Loaches of the subfamily nemacheilinae in China. Jiangsu Science and Technology Publishing House, Nanjing 